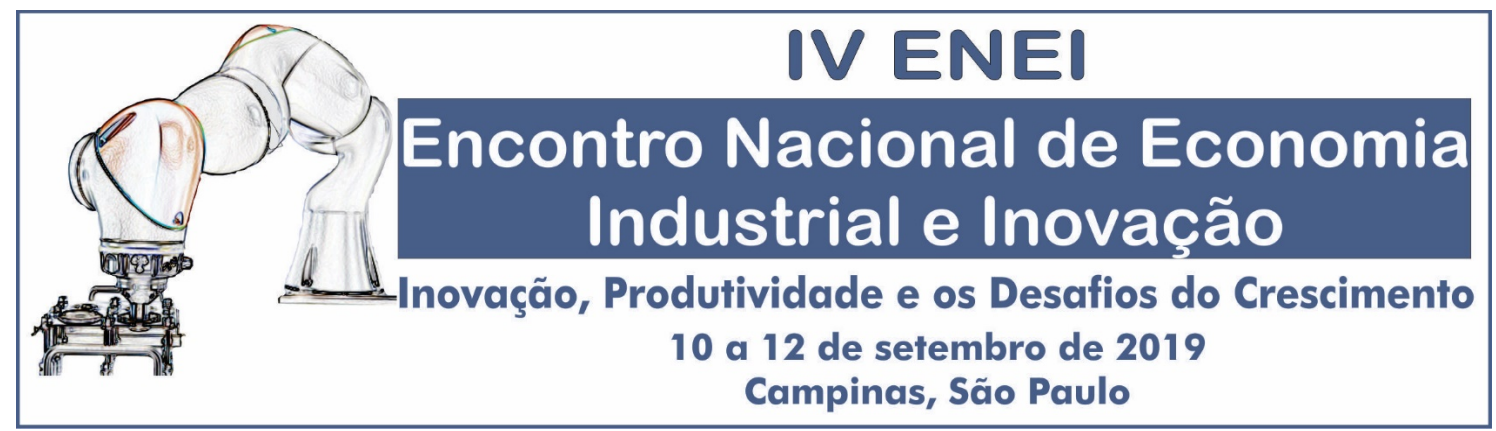

PROPOSTA DE MODELO PARA RESSUPRIMENTO DE ESTOQUES NO PROCESSO MRP COM OPÇÃO DE TRANSFERÊNCIAS: UMA APLICAÇÃO DE PROGRAMAÇÃO LINEAR EM UM CONTEXTO MULTI-FÁBRICAS

Área temática: 5.4 Mudanças técnicas, organizações e instituições

Classificação Journal Economic Literature - C61 - Técnicas de otimização; Modelos de programação; Análise dinâmica

Murilo José Rosa ${ }^{1}$

murilorosa.usp@gmail.com

Geciane Silveira Porto ${ }^{1}$

geciane@usp.br

${ }^{1}$ Faculdade de Economia, Administração e Contabilidade de Ribeirão Preto (FEA-RP/USP)

Av. Bandeirantes, 3900 - Campus da USP, Ribeirão Preto - SP, 14040-900 


\title{
PROPOSTA DE MODELO PARA RESSUPRIMENTO DE ESTOQUES NO PROCESSO MRP COM OPÇÃO DE TRANSFERÊNCIAS: UMA APLICAÇÃO DE PROGRAMAÇÃO LINEAR EM UM CONTEXTO MULTI-FÁBRICAS
}

\section{Resumo}

Esse artigo propõem um modelo alternativo para a tratativa da situação de excesso de estoques nas organizações, que podem acontecer em razão de variabilidades esperadas na dinâmica de gestão de MROs. O modelo se encaixa dentro do processo MRP como um componente de apoio na decisão entre compras ou transferências de estoques no contexto multi-fábricas, apoiado na premissa de otimização do ressuprimento. $\mathrm{O}$ modelo proposto foi aplicado em uma organização do setor sucroenergético, durante um período de 9 meses, com resultados de transferência que somam aproximadamente de $\mathrm{R} \$ 3,8$ milhões em valor de inventário.

Palavras-chave: otimização do ressuprimento; transferência de estoques; processo MRP.

\section{REPLENISHMENT MODEL PROPOSAL FOR MRP PROCESS WITH TRANSFER OPTION: A LINEAR PROGRAMMING APPLICATION IN A MULTI-FACTORY CONTEXT}

\begin{abstract}
This article aims to propose an alternative model for a situation of inventory excess in organizations, which can occur due to the expected variabilities in the dynamics of MROs management. This model is part of the MRP process as a supporting component in the choice between purchase or inventory transfer in a multi-factory context, supported by the premise of replenishment optimization. For a period of nine months the model was applied in a sugarcane environment with results of about BRL 3.8 million in inventory value.
\end{abstract}

Keywords: replenishment optimization; inventory transfer; MRP process. 


\section{INTRODUÇÃO}

A literatura para modelos de planejamento e gestão de estoques tipicamente assume balanceamentos perfeitos sob a lógica do MRP (Material Requirement Planning). Na prática, os parâmetros calculados acabam sendo aplicados, contudo a complexidade das operações e o comportamento muitas vezes imprevisível das diversas variáveis da cadeia de abastecimento (e.g. lead time de transporte e taxa de demanda) impõe um resultado diferente do ideal. Excessos ou faltas de estoque são situações prováveis dentro dessa dinâmica (Bailey e Helms, 2007).

A forma com que as organizações tratam essa questão apresenta variações conforme o caso. Alguns simplesmente privilegiam o nível de serviço de suas operações e adotam estratégias conservadoras, como por exemplo, aumentando o estoque de segurança. As principais consequências dessa opção são o alto comprometimento do fluxo de caixa, o custo de carregamento e o risco de obsolescência dos materiais em estoque (Rego e Mesquista, 2011). Outras organizações preferem manter os "cálculos originais" do balanceamento e então administrar pontualmente os casos de ruptura. Essa opção, normalmente traz uma carga de stress organizacional em que a falta ou excesso de estoque gera conflitos entre áreas internas ou entre organizações, principalmente a montante da cadeia de suprimento (Alfieri et al. 2017). Em qualquer caso, a escolha deve ser ponderada em função do trade-off inerente na tomada de decisão.

Uma abordagem alternativa para essa questão é trabalhar a possibilidade de transferência dos materiais em excesso, quando a organização possui mais de uma fábrica, filial ou unidade de negócio. Dessa forma, o eventual excesso de estoque não fica estacionado nas prateleiras dos almoxarifados, servindo como "oferta" durante o processo de análise de ressuprimento conduzido dentro do processo MRP. Nesse estudo entende-se como excesso o volume de inventário exatamente superior ao previsto em política de material vigente.

Haja vista o contexto apresentado, esta pesquisa tem por objetivo principal propor e aplicar um modelo em programação linear - adaptado a partir do problema clássico de transporte introduzido de forma pioneira por Hitchcock (1941) e Koopmans (1949) - que visa otimizar a posição de estoques por meio da minimização dos custos de aquisição de material e custos logísticos. Esse modelo é adaptado para um cenário de multi-fábricas em que: 
a) Há similaridade na utilização de materiais MRO (Maintenance, Repair and Operations) com demanda independente (i.e., as fábricas têm as mesmas tecnologias e utilizam os mesmos materiais);

b) Existe capacidade efetiva de organizar e executar a logística de transporte (transferências) entre unidades de negócio com fretes fracionados dentro do período entre os processamentos do MRP.

Trata-se aqui o estoque dentro de uma sistemática em que o volume global é equalizado considerando a contribuição de cada unidade de negócio (fábrica) no resultado da organização. Decisões tomadas com base em visões sistêmicas buscam evitar resultados isolados que "subotimizam" o potencial resultado global.

A principal contribuição empírica do modelo constitui-se em sua utilização combinada com o processamento do MRP, no momento subsequente as análises de demanda em que são dispostas as opções de fonte de suprimento. Do ponto de vista teórico, o modelo avança na discussão sobre o tratamento de excessos de estoque nas organizações multi-fábricas, endereçando com matemática aplicada a situação das decisões entre compras e ou transferências.

Destaca-se que o modelo proposto foi aplicado em uma organização agroindustrial sucroenergética durante o período de nove meses. Os resultados dessa aplicação, discutidos na seção 3, além de indicar a sua viabilidade, trazem algumas lições aprendidas durante o projeto de implantação, que nos permitiram melhorar ou repensar o modelo.

Este é um estudo com abordagem quali-quanti por adotar métodos mistos para o atingimento dos objetivos. (Sampieri et al. 2006) que se caracteriza pela utilização do método de pesquisa-acão (Thiollent, 1992), no qual há inserção do pesquisador no universo analisado propondo soluções e ou alterações na realidade afim de contribuir com melhorias. $\mathrm{O}$ estudo envolveu uma participação direta na proposição do modelo e ao longo dos nove meses de acompanhamento da sua implementação e ajustes para o seu adequado funcionamento. Do ponto de vista quantitativo, há a aplicação de pesquisa operacional com a abordagem do modelo pautada em programação linear.

Este artigo está estruturado em 4 seções, sendo a primeira parte reservada à introdução ao tema. Na segunda seção apresenta-se a revisão bibliográfica dos modelos de gestão organizacional para estoque, em particular aqueles ligados a ressuprimento. $\mathrm{Na}$ seção três são apresentados os 
resultados do artigo, com a proposta de modelo. Por fim, na última seção são feitas as considerações finais.

\section{O ESTOQUE NA GESTÃO DAS ORGANIZAÇÕES}

Slack (et al. 2009) mostra que uma redução de 5\% nas despesas anuais com materiais é capaz de proporcionar um incremento de até 35\% no lucro de uma organização. Esse benefício exponencial justifica o trabalho, acadêmico e gerencial, com foco em melhorar a eficiência em toda a cadeia de abastecimento.

Em termos contábeis e financeiros, os estoques representam ativos circulantes e despesas orçamentarias que demandam atenção da Organização. Como forma de controlar a dinâmica de materiais, as empresas se atentam (i) ao giro de inventário para medir o nível de imobilização do capital e risco de ressuprimento (Lee et al. 2015) e (ii) as demonstrações financeiras e contábeis para analisar os indicadores relacionados à parcela do ativo (Feng et al. 2015).

Para ambientes com demanda predominantemente independente, familiares ao contexto MRO, a gestão tende a ser menos precisa, pois os gestores devem utilizar informações históricas ou devem confiar na expertise dos usuários para direcionar o processo de ressuprimento.

A situação de excesso de estoque ocorre por intermédio de quatro fatores previamente identificados na literatura (Rego e Mesquita, 2011):

- Compra sem histórico de consumo: quando um material é novo na atividade operacional e não há qualquer histórico que indique a taxa de consumo aproximada. Com isso é necessário estimar um consumo futuro para subsidiar o processo de compra e abastecimento. Esse volume pode estar superestimado, ocasionando sobras.

- Atualização da política de material: nos casos particulares em que as necessidades de determinado material são atualizadas a níveis inferiores, descomprometendo o saldo de estoque com a operação local de uma unidade de negócio.

- Retirada da política de material: em situações que determinado material passa a não ter mais parâmetros de política, como por exemplo, quando há identificação de obsolescência técnica.

- Tamanhos de lote superiores à necessidade: quando não é possível conciliar o tamanho do lote calculado na política de estoque ao tamanho de lote negociado com fornecedores no mercado. 


\subsection{Modelos de ressuprimento na cadeia de suprimentos}

Existem alguns modelos tradicionais de ressuprimento que auxiliam as Organizações na tarefa de abastecer os estoques. O modelo MRP, concebido no começo dos anos 60 e aperfeiçoado no Sistema de Apoio a Produção MRP II e depois no ERP, é amplamente adotado na manufatura e possibilita o atendimento de diversos tipos de organização (Rabinovich e Evers, 2002). Originalmente o modelo buscava atender a operação a partir da lista técnica do produto acabado, respeitando os lead times parametrizados em cada ordem de produção ou compra e usualmente liberando as etapas por meio da "programação de traz para frente" (backward schedulling). A partir da consideração de diferentes comportamentos de demanda, o modelo MRP evoluiu e ofereceu opções para materiais com consumos estocásticos, esporádicos e independentes. O universo de políticas de materiais disponíveis também foi expandido e as possibilidades de configuração ampliadas (Hautaniemi e Pirttila, 1999).

Em contraponto ao MRP, que valoriza o processamento computacional (Gupta e Snyder, 2009), o modelo proposto pelo Just in Time, no final dos anos 60, surgiu como uma opção mais intuitiva para o ressuprimento. Nele, o estoque de peças da fábrica é reduzido (e no limite eliminado) como parte de uma filosofia para que os problemas operacionais possam aparecer e enfim serem tratados. Nesse caso os custos logísticos do processo de ressuprimento assumem protagonismo, pois o tamanho do lote, sensível a "puxada da produção" (kanban), torna-se menor fazendo com que as frequências de abastecimento sejam maiores (Vonderembse et al. 1995).

Pode-se considerar também o modelo colaborativo de previsões (Collaborative Planning, Forecasting, and Replenishment - CPFR) como um mecanismo de integração entre empresas capaz de reduzir a incerteza das Operações e consequentemente a incerteza ligada à manutenção de estoques (Panahifar et al. 2015). Tal modelo pode servir como base para um processo de compartilhamento de informações de PCP (Planejamento e Controle da Produção) que venha a auxiliar o planejamento de ressuprimento da cadeia produtiva.

Versões mais atuais para essa tarefa (de ressuprimento) avaliam modelos que buscam a visão sistêmica da cadeia de suprimentos (Fattahi et al. 2014). Nestes, o estoque da cadeia como um todo é otimizado em detrimento a situação ótima de cada um dos elos participantes (otimização 
multi-echolon). Outros modelos de otimização, adaptados a contextos específicos, também se propõe a ajudar o ressuprimento eficiente dos estoques. Taleizadeh et al. (2015) apresentam modelo integrado para ambientes com itens perecíveis utilizando Vendor Managed Invetory (VMI); Tempelmeie e Bantel (2015) estudam o processo de ressuprimento integrando os elementos de estoque de segurança e capacidade de transporte; Grewal et al. (2015) apresentam diferentes estratégias para realizar o abastecimento quando a demanda é sazonal; por fim, Orbegozo et al. (2018) traz uma revisão atualizada dos modelos de ressuprimento integrados ao planejamento da produção com os algoritmos propostos e utilizados pelos pesquisadores no assunto.

\section{ANALISE E DISCUSSAO DOS RESULTADOS}

$\mathrm{Na}$ análise e discussão dos resultados estão as seções que apresentam o modelo proposto, que mostram a aplicação feita desse modelo no contexto sucroenergético e por fim são feitas as argumentações sobre a contribuição desse modelo para gestores e pesquisadores.

\subsection{A construção do modelo proposto}

Com o objetivo de minimizar o custo total da operação de abastecimento dos estoques, montou-se uma função objetivo para o modelo em programação linear. Essa função avalia qual opção (ou combinação de opções) é mais econômica para atendimento da demanda gerada no MRP, partindo de três fontes: estoque disponível, estoque transferido e estoque comprado de fornecedores.

Função objetivo:

$\mathrm{Z}=\sum_{\mathrm{i}=1}^{\mathrm{m}} \sum_{\mathrm{j}=1}^{\mathrm{z}} \sum_{\mathrm{h}=1}^{\mathrm{n}}\left(\operatorname{COST}_{-} \operatorname{TRANSF}_{(\mathrm{i}, \mathrm{j}, \mathrm{h})} \cdot \mathrm{TX}_{-} \mathrm{OCUPA}_{(\mathrm{h})}\right) \cdot \mathrm{QTD}_{-} \operatorname{TRANSF}_{(\mathrm{i}, \mathrm{j}, \mathrm{h})}+1.05 \sum_{\mathrm{j}=1}^{\mathrm{z}} \sum_{\mathrm{h}=1}^{\mathrm{n}} \operatorname{COST} \_B U Y_{(\mathrm{j}, \mathrm{h})} \cdot \mathrm{QTD}_{-} \mathrm{BUY}_{(\mathrm{j}, \mathrm{h})}+\sum_{\mathrm{j}=1}^{\mathrm{z}} \sum_{\mathrm{h}=1}^{\mathrm{n}} \operatorname{COST}_{-} \mathrm{FREE}_{(\mathrm{j}, \mathrm{h})} \cdot \mathrm{QTD}_{-} \mathrm{FREE}_{(\mathrm{j}, \mathrm{h})}$

Sujeito as seguintes restrições: 


$$
\begin{aligned}
& \sum_{\mathrm{i}=1}^{\mathrm{m}} \text { QTD_TRANSF }_{(\mathrm{i}, \mathrm{j}, \mathrm{h})}+\text { QTD_BUY }_{(\mathrm{j}, \mathrm{h})}+\text { QTD_FREE }_{(\mathrm{j}, \mathrm{h})}=\text { DEMAND }_{(\mathrm{j}, \mathrm{h})} \quad \forall \mathrm{j}, \mathrm{h} \\
& \sum_{\mathrm{j}=1}^{\mathrm{z}} \operatorname{QTD}_{-} \operatorname{TRANSF}_{(\mathrm{i}, \mathrm{j}, \mathrm{h})} \leq \operatorname{OFFER}_{(\mathrm{i}, \mathrm{h})} \quad \forall \mathrm{i}, \mathrm{h} \\
& \operatorname{OFFER}_{(\mathrm{i}, \mathrm{h})}=\operatorname{SUPPLY}_{(\mathrm{i}, \mathrm{h})}-\left(\text { PREV_CONS }_{(\mathrm{i}, \mathrm{h})}+\text { DEMANDORIG }_{(\mathrm{i}, \mathrm{h})}\right) \\
& \text { QTD_FREE }_{(\mathrm{j}, \mathrm{h})} \leq \text { AVAIB_FREE }_{(\mathrm{j}, \mathrm{h})}
\end{aligned}
$$

As restrições à função objetivo limitam o modelo, pois:

- Permitem que a demanda seja atendida por intermédio de três variáveis: (i) quantidade em transferência, (ii) quantidade em compra e (iii) quantidade disponível no estoque da própria unidade de negócio;

- Garantem que as quantidades transferidas não sejam maiores que aquelas reservadas para tal finalidade;

- Configuram a oferta disponível de material, poupando o estoque necessário para o uso da própria unidade de negócio.

- Asseguram que o estoque próprio utilizado seja somente aquele que está efetivamente disponível.

Onde:

$\mathrm{i}=$ Unidade de origem $\mathrm{i}$;

$\mathrm{J}=$ Unidade de destino $\mathrm{j}$;

$\mathrm{h}=$ Material h;

COST_TRANSF $=$ custo de transferência entre unidades de negócio;

TX_OCUPA = taxa de ocupação veicular;

QTD_TRANSF = quantidade para transferir (variável de decisão);

COST_BUY = custo de compra dos materiais;

QTD_BUY = quantidade para comprar (variável de decisão);

DEMAND = necessidade de material (visão da unidade de destino);

SUPPLY = materiais disponíveis em estoque; 
PREV_CONS = previsão de consumo;

DEMANDORIG = necessidade de material (visão da unidade de origem);

COST_FREE = "Custo zero" do aproveitamento do estoque disponível;

QTD_FREE = Quantidade utilizada da própria unidade (variável de decisão);

AVAIB_FREE $=$ Estoque disponível na unidade de origem.

As premissas utilizadas na estruturação do modelo foram:

- Há $100 \%$ de correspondência entre estoque físico e contábil das unidades de negócio. Isto é, não foi considerada qualquer existência de "taxa de quebra" no estoque contábil.

- Os tempos de criação de requisição de compra, processo de negociação com fornecedores, criação de pedidos de compra, aprovações sistêmicas de workflow, lead time de transporte, entre outros, não foram considerados no modelo.

- Na parametrização de ocupação dos materiais, foi definido transporte por meio de caminhão modelo truck com capacidade de carga na ordem de 15 toneladas. Foi também atribuído um coeficiente para cada material que representa sua parcela de ocupação nesse veículo $(0.01 \% \leq$ coeficiente $\leq 100 \%)$.

- Na definição do preço de frete entre unidades de negócio, considerou-se uma parcela de custo fixo de BRL 400,00 mais um custo variável de BRL 2,40/km.

- O preço de compra de determinado material é igual ao preço desse mesmo material no estoque da unidade de destino acrescido $5 \%$ (preço multiplicado pelo escalar 0,05 ) para efeito estimado de marcação a mercado.

- A unidade de origem deve disponibilizar (para transferência) somente a quantidade de determinado material que exceda sua política mais a previsão de consumo para o próximo mês (com base em consumo médio histórico). Essa premissa faz com que haja cobertura de estoque suficiente para mitigar as ocorrências de transferência e posterior compra para a mesma fábrica no curto/curtíssimo prazo.

A coleta dados e preparação dos parâmetros foram feitas da seguinte forma:

- O sinal de demanda veio da lógica MRP, com os cálculos da análise de ressuprimento oferecidos em relatório extraído diretamente no ERP da Organização. 
- Os volumes acima da política de estoque foram imediatamente reservados e classificados como "oferta" para transferência. Essa atividade foi feita por intermédio de planilha eletrônica.

- As informações sobre ocupação dos materiais foram construídas pela área especializada na gestão dos estoques. Cada material foi devidamente analisado com base em suas dimensões e pesos de tal forma que foi possível atribuir uma taxa de ocupação como referência para transporte fracionado.

- O custo de transferência foi obtido através das distâncias rodoviárias "porta-a-porta" com a coleta de quilometragem no aplicativo online Google Maps.

- O custo de compra foi composto a partir da extração do relatório de estoque no dia em que cada análise de ressuprimento foi realizada. Esse valor é resultado de uma lógica de custo médio a partir da entrada de cada material no estoque.

- A previsão de consumo foi estimada considerando a média de consumo mensal nos últimos dois anos para cada material (2015 e 2014), retirando meses de pico em função de características de sazonalidade do negócio.

Para processar o modelo em programação linear foi utilizado o software GAMS (General Algebraic Modeling System) com algoritmo CPLEX (Simplex com linguagem C) e interface Excel com GDX (GAMS Data Exchange). O tempo médio de processamento foi de 32 segundos por rodada de MRP. As informações de entrada do GAMS encontram-se no tópico de apêndice do artigo.

\subsection{Aplicação em ambiente agroindustrial sucroenergético}

O modelo foi aplicado em uma organização que está inserida em um ambiente agroindustrial de produtos sucroenergéticos. A mesma conta com onze unidades de negócio distribuídas em cinco Estados brasileiros e tem faturamento anual de aproximadamente R\$ 6 bilhões. Em se tratando de gestão dos estoques nessa organização, vale ressaltar que:

- Cada unidade tem uma estrutura e equipe de almoxarifado que executa as atividades operacionais de recebimento, armazenagem e entrega de materiais. Há em cada unidade um coordenador responsável pelo processamento do MRP. 
- Há uma equipe corporativa para questões tático-estratégicas, responsável por auxiliar os almoxarifados na definição de políticas e liderar as iniciativas de projetos.

- A empresa utiliza o MRP de um software ERP (Enterprise Resource Planning), com os benefícios intrínsecos da integração do módulo de gestão de materiais com os demais módulos de gestão da empresa.

Em função de definições internas a própria organização classificou quais unidades estariam habilitadas para transferência de materiais. Essa configuração levou em conta questões geográficas, contábeis, fiscais e operacionais. As unidades que iriam transferir materiais entre si foram aglomeradas em três clusters (Figura 1).

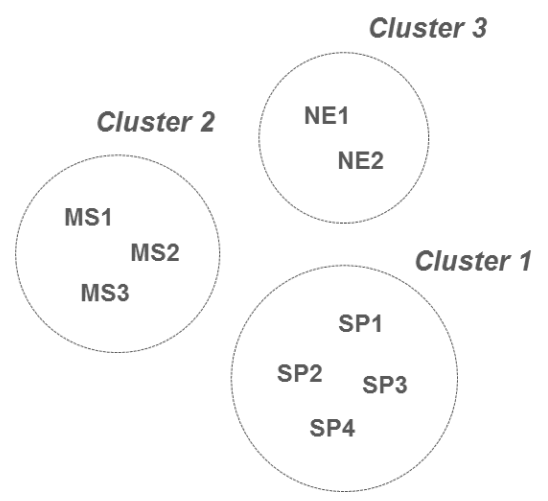

Figura 1. Ilustração dos clusters com as unidades habilitadas para transferência.

Fonte: elaborada pelos autores.

De suas onze unidades de negócio somente duas ficaram a margem do modelo de transferência por não se enquadrarem nas características de nenhum dos três clusters criados inicialmente.

O processo mais amplo de ressuprimento (Figura 2) foi montado na organização a partir de um fluxo periódico, expresso no calendário de processamento do MRP (rodadas de MRP). Após isso, combinaram-se (entre os participantes) os dias da semana em que cada parte do processo iria acontecer. Nesse caso começava-se sempre na quarta-feira de cada semana com a geração da demanda por meio do processamento do MRP. Nessa fase deduz-se da demanda bruta o estoque na unidade, suas requisições de compra em andamento e os saldos a fornecer dos pedidos de compra que estão compartilhados com fornecedores. O saldo final dessas deduções é a demanda líquida que vai para a próxima etapa do processo. 
De posse das saídas de MRP, o modelo de transferência era aplicado nas quintas-feiras. Toda a análise era feita de forma centralizada, pela equipe corporativa, já que havia a necessidade de uma visão global do processo. Nas sextas-feiras os saldos das ordens gravadas no MRP eram corrigidos pelas transferências e por fim liberavam-se as necessidades de compra para as aprovações sistêmicas do workflow empresarial.

$\mathrm{Na}$ semana seguinte, entre o domingo e a terça-feira, executavam-se em paralelo as atividades de compra e transferência dos materiais entre os almoxarifados. Era essencial que essas etapas acontecessem impreterivelmente antes das quartas-feiras para que o processamento da próxima rodada do MRP não sofresse com um "overlap" de dados, contaminando assim os novos resultados.

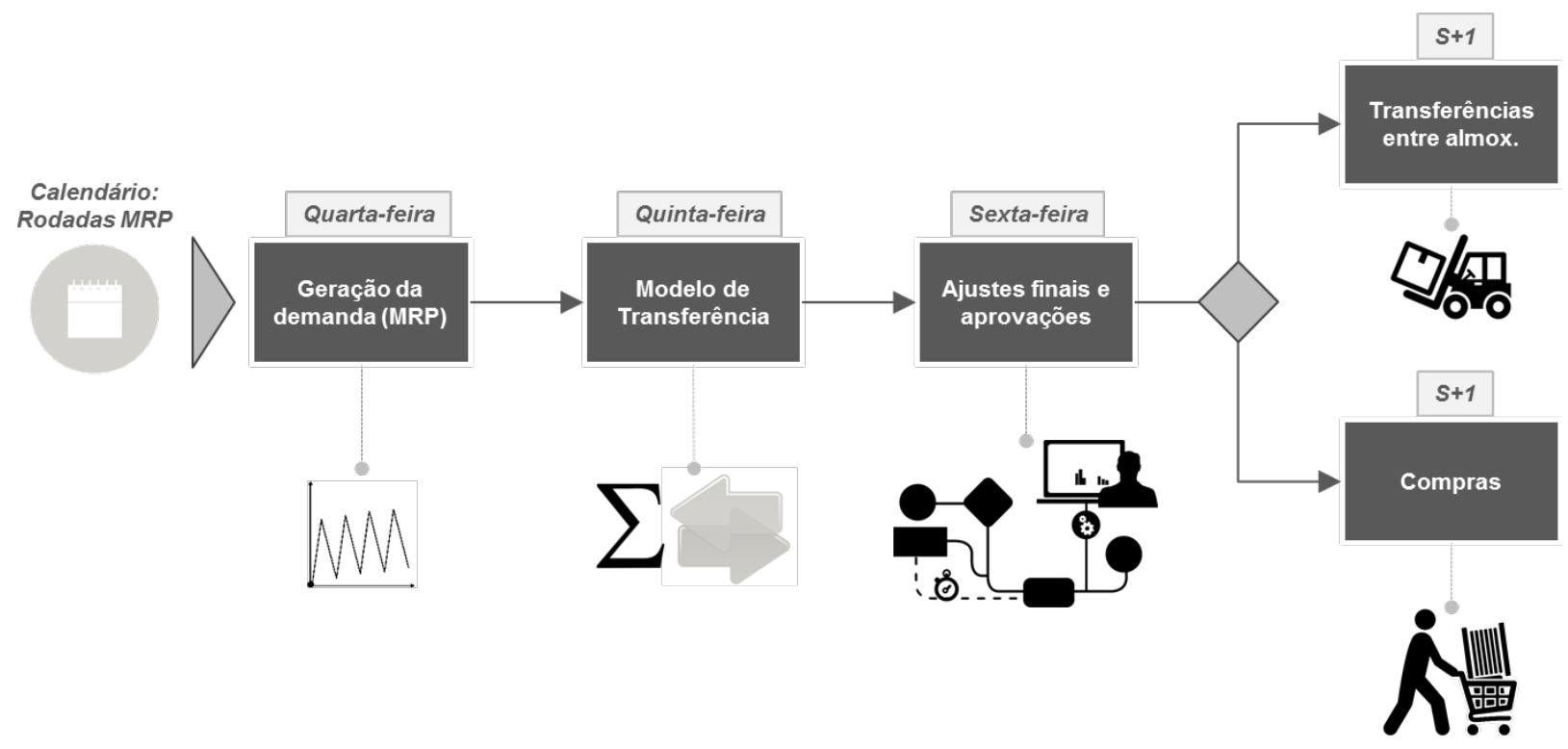

Figura 2. Processo de ressuprimento periódico com a integração do modelo de transferência.

Fonte: elaborada pelos autores.

O processo começou em março de 2016, e entre os meses de março até agosto o processo ocorria em bases quinzenais. Entre agosto até e novembro adotou-se processamento semanal para o MRP.

\subsection{Contribuições do modelo proposto}


A organização objeto da aplicação do modelo conta com um estoque médio de cerca de R\$ 30 milhões para os materiais que integram a lógica MRP. Esse valor representava aproximadamente $26 \%$ do estoque total da empresa. Todos os itens tinham o mesmo tipo de política de estoque, com Ponto de Ressuprimento e Tamanho de Lote $(\mathrm{R}, \mathrm{Q})$, sendo a reposição até o máximo. O restante dos itens ( $74 \%$ que não estavam em MRP) apresentavam demanda dependente, tinham taxa de demanda esporádica, eram reservas técnicas ou ainda estavam em estudo sobre os parâmetros ideais a serem configurados no MRP.

Ao final dos noves meses de utilização do modelo, o resultado acumulado em transferências foi de R \$ 3,8 milhões (Figura 3). Durante esse período o MRP foi processado vinte e cinco vezes. Não obstante, ocorreu um efeito múltiplo com a transferência de materiais. Além de aproveitar o estoque disponível, que eventualmente poderia se transformar em estoque com giro lento na análise de inventário, deixou-se de acessar o processo de compras da organização. Com isso, exige-se menos do fluxo de caixa, liberando capital para outros investimentos de interesse.

\begin{tabular}{|c|c|c|}
\hline & Em BRL & \\
\hline Rodadas 01 a 05 & 543.742 & \\
\hline Rodadas 06 a 10 & 950.869 & \multirow{3}{*}{$\begin{array}{l}\text { Efeito múltiplo (2x): } \\
\text { - Deixa-se de comprar } \\
\text { - Utiliza-se o estoque disponivel }\end{array}$} \\
\hline Rodadas 11 a 15 & 904.911 & \\
\hline Rodadas 16 a 20 & 556.172 & \\
\hline Rodadas 21 a 25 & 855.139 & \multirow[t]{2}{*}{$\mathbf{4}$} \\
\hline Total & 3.810 .833 & \\
\hline
\end{tabular}

Figura 3. Resultados monetários das transferências de materiais após as rodadas de MRP.

Fonte: elaborada pelos autores.

A primeira rodada de MRP coincidiu com a implantação da política de estoques na organização. Com efeito, existia a expectativa entre os participantes do projeto de que haveria uma carga significante de atualizações das políticas de MRP conforme fossem realizadas as operações de ressuprimento no curto e médio prazo. Isso foi confirmado e gerou várias etapas de projeto para alinhamento entre taxa de demanda e parâmetros calculados, gerando ainda mais valor agregado ao modelo com a opção de transferir os estoques excedentes.

Além dos resultados obtidos em rodadas de MRP, o modelo mostrou-se útil para outros momentos de demanda de materiais na organização agroindustrial estudada. Por exemplo, durante 
as manutenções pesadas de entressafra a aplicação trouxe resultados ainda mais expressivos com economias na ordem de R \$ 5,9 milhões e R \$ 1,3 milhões nos anos de 2015 e 2016, respectivamente.

A expectativa das equipes envolvidas está na atualização do modelo a partir das oportunidades de melhoria identificadas (comentadas no tópico a seguir). Além disso, espera-se continuar utilizando-o de forma recorrente somente em meses de consumo regular de materiais. Exatamente os mesmos em que há rodada de MRP com materiais de MRO.

Os resultados da aplicação desse modelo endereçam justamente os pontos da salientados no trabalho de Rego e Mesquista et al. (2001) que tratam da dificuldade em gerenciar estoques de MRO, frente a diversidade de variáveis que impactam o nível do inventário.

\section{CONSIDERAÇÕES FINAIS}

Esta pesquisa se propôs a desenvolver e aplicar um sistema de apoio à decisão que capturasse as oportunidades em ambientes multi-fábrica com excesso de estoque. O que se mostrou bastante promissor uma vez que após nove meses de uso em uma organização agroindustrial os resultados somaram o montante de R \$ 3,8 milhões em transferências. Esse impacto pode ser considerado dentro de um universo de gestão de materiais de cerca de $\mathrm{R} \$ 30$ milhões.

Como lição aprendida durante a implantação, destaca-se que com o uso de apresentações com infográficos e explicações básicas do problema, foi possível obter uma aceitação razoável para a implantação do projeto, reduzindo significativamente a aversão/resistência a mudança que em geral ocorrem. Deve-se considerar que a organização que se sujeitou ao modelo não tinha nenhuma outra aplicação de programação linear (em qualquer departamento) ou iniciativa parecida em andamento e aceitou o desafiou de implementar esta inovação organizacional.

A forma de comunicar os resultados a cada rodada de MRP foi um aspecto crítico para o sucesso. Já que cada responsável por converter as requisições de compra ou organizar o transporte de transferência precisava entender exatamente seu papel no processo. Para que isso acontecesse, foi necessário treinar as equipes envolvidas e criar um grupo de apoio capacitado para resolução de dúvidas ou problemas pontuais. 
O modelo aplicado foi considerado, pela equipe de implantação, como um passo introdutório para o aperfeiçoamento da atividade de transferência de estoques, muito embora seus resultados já tenham sido expressivos durante os primeiros meses em uso. Cabe salientar que anteriormente a essa aplicação a Organização estudada não realizava qualquer tipo de análise de transferência sobre os estoques de forma periódica. Existia somente uma análise dos itens da curva A e B (curva $\mathrm{ABC}$ ) durante o período de entressafra que durava entre 3 a 4 semanas e que após a aplicação do modelo proposto passou a ocorrer para $100 \%$ do estoque, durando em média 3 dias.

Sobre as limitações desse trabalho é preciso destacar:

- Não houve utilização de uma lógica que habilita a transferência de materiais a partir de uma consolidação de volumes. Tal consideração iria de encontro com a premissa de frete fracionado e traria uma nova premissa de viabilidade financeira para transporte próprio ou de carga fechada.

- Não utilização de restrições para compra de material a partir do tamanho de lote usualmente negociado no mercado (e.g. Embalagens econômicas).

- Desconsideração de variável para o fator de custo financeiro com a imobilização de estoque. No modelo proposto o custo de carregamento dos estoques não foi uma variável que influenciava as variáveis de decisão.

- Não utilização de parâmetros fiscais para aproveitamento de novas oportunidades de transferência, principalmente em transportes de materiais entre unidades localizadas em Estados distintos.

- Não utilização de cenários com opção de entrepostos logísticos.

- Não foi feito qualquer tipo de análise “e-se” (“what-if”) com o relaxamento de uma ou mais premissas adotadas.

Uma última limitação importante do estudo está no fato que o modelo foi desenvolvido para um setor em particular, assim a priori não se pode esperar que os resultados deste modelo sejam exatamente iguais e replicáveis para outros setores. Desta forma, em futuros estudos, pretende-se aplicar este modelo para industrias de diferentes setores, desenvolvendo a calibração necessária no modelo para as respectivas características das diferentes industrias.

Ressalta-se pôr fim a importância de um cadastro de materiais saneado, já que é possível perder oportunidades de transferência quando a correspondência entre SKUs (Stock Keeping Units) não é 
precisa. Isto é, ocorrências de duplicidade em base cadastral imprimem um impacto negativo nesse processo.

\section{Referências}

ALFIERI, A; PASTORE, E; ZOTTERI, G. Dynamic inventory rationing: How to allocate stock according to managerial priorities. An empirical study. International Journal of Production Economics. Volume 189, July 2017, Pages 14-29.

BAILEY, G.J; HELMS, M. M. MRO inventory reduction-challenges and management: a case study of the Tennessee Valley Authority. Production Planning \& Control: The Management of Operations, 18:3, 261-270, DOI: 10.1080/09537280601127351.

FATTAHI, M; MAHOOTCHI, M; MOATTAR HUSSEINI, S.M; KEYVANSHOKOOH, E; ALBORZI, F. Investigating replenishment policies for centralised and decentralised supply chains using stochastic programming approach. International Journal of Production Research. 2014; DOI: 10.1080/00207543.2014.922710.

FENG, M; LI, C; McVAY, S. E; SKAIFE, H. Does Ineffective Internal Control over Financial Reporting affect a Firm's Operations? Evidence from Firms' Inventory Management. The Accounting Review: March 2015, Vol. 90, No. 2, pp. 529-557.

GREWAL, C. S; ENNS, S. T; ROGERS, P. Dynamic reorder point replenishment strategies for a capacitated supply chain with seasonal demand. Computers \& Industrial Engineering. Volume 80, February 2015, Pages 97-110

GUPTA, M; SNYDER, D. Comparing TOC with MRP and JIT: a literature review. International Journal of Production Research. Volume 47, 2009 - Issue 13.

HAUTANIEMI, P; PIRTTILA, T. The choice of replenishment policies in an MRP environment. International Journal of Production Economics. Volume 59, Issues 1-3, March 1999, Pages 8592.

HITCHCOCK. F.L. Distribution of a product from several sources to numerous localities. Journal of Mathematics and Physics. 1941; 20; 224-230. 
KOOPMANS, T.C. Optimum utilization of the transportation system. Econometrica; 1949; 17; 34.

LEE, H; ZHOU, J; HSU, P. The role of innovation in inventory turnover performance. Decision Support Systems. Volume 76, August 2015, Pages 35-44.

ORBEGOZO A; ANDRES B; MULA J; LAURAS M; MONTEIRO C; MALHEIRO M. (2018) An Overview of Optimization Models for Integrated Replenishment and Production Planning Decisions. In: Viles E., Ormazábal M., Lleó A. (eds) Closing the Gap Between Practice and Research in Industrial Engineering. Lecture Notes in Management and Industrial Engineering. Springer, Cham.

PANAHIFAR P. BYRNE P.J. CATHAL HEAVEY. A hybrid approach to the study of CPFR implementation enablers, Production Planning \& Control, 26:13, (2015) 1090-1109, DOI: 10.1080/09537287.2015.1011725

RABINOVICH, E; EVERS, P. T. Enterprise-wide adoption patterns of inventory management practices and information systems. Transportation Research Part E 38 (2002) 389-404.

REGO, J. R; MESQUITA, M. A. Controle de estoque de peças de reposição: uma revisão da literatura. Produção, v. 21, n. 4, out./dez. 2011, p. 645-655.

SAMPIERI, R.H.; COLLATO, C.F.; LUCIO, P.B.Metodologia de Pesquisa. 3a. Edição. São Palo:McGraw-Hill, 2006.

SLACK, N; CHAMBERS, S; HARLAND, C; HARRINSON, A; JOHNSTON, R. Administração da Produção. Edição compacta. São Paulo: Atlas, 1999.

TEMPELMEIER H; BANTEL O. Integrated optimization of safety stock and transportation capacity. European Journal of Operational Research. Volume 247, Issue 1, 16 November 2015, Pages 101-112.

THIOLLENT, M. Metodologia da Pesquisa-ação. 14a. edicão, Editora Cortez, 1992.

VONDEREMBSE, M; TRACEY, M; LENG TAN, C; BARDI, E.J. Current purchasing practices and JIT: some of the effects on inbound logistics, International Journal of Physical Distribution \& Logistics Management, Vol. 25 Iss 3 pp. 33 - 48. (1995). 


\section{Apêndice 01:}

Arquivo com a programação de entrada no software GAMS:

\section{SETS}

i Unidade de origem

j Unidade de destino

h Material;

\section{PARAMETERS}

COST_TRANSF $(\mathrm{i}, \mathrm{j}, \mathrm{h})$ Custo da transferência entre a unidade de origem i e destino $\mathrm{j}$ do material $\mathrm{h}$

COST_BUY (j,h) Custo da compra do material h para a unidade de destino $\mathrm{j}$

DEMAND $(\mathrm{j}, \mathrm{h})$ Necessidade do material $\mathrm{h}$ na unidade de destino $\mathrm{j}$

DEMANDORIG $(\mathrm{i}, \mathrm{h})$ Demanda na visão origem

SUPPLY (i,h) Quantidade disponível de estoque do material h na origem i

TX_OCUPA (h) Taxa de ocupação do material h

PREV_CONS (i,h) Previsão de consumo do material h na unidade de origem i

AVAIB_FREE (j,h) Volume já disponível de estoque do material h no destino j

\$CALL GDXXRW.EXE otimizacao_estoque.xls Index=leitura!a2

\$GDXIN otimizacao_estoque.xls

\$LOAD i j h COST_TRANSF COST_BUY DEMAND DEMANDORIG SUPPLY TX_OCUPA

PREV_CONS AVAIB_FREE

\$GDXIN

\section{VARIABLES}

QTD_TRANSF (i,j,h) Quantidade do material h transferido da unidade i para a unidade j

QTD_BUY (j,h) Quantidade do material h comprado para a unidade $\mathrm{j}$

Positive variables QTD_TRANSF, QTD_BUY; 


\section{EQUATIONS}

MIN_CUSTO_TOTAL Calcula as quantidades de transferência e compra que minimizam o custo ATENDIMENTO Garante que as quantidades transferidas mais as quantidades compradas sejam iguais as quantidades demandadas

RESTR_SUPPLY Garante que a quantidade transferida seja igual ou menor que a quantidade disponível em estoque mais a previsão de consumo

USO_STOCK Uso do estoque disponível

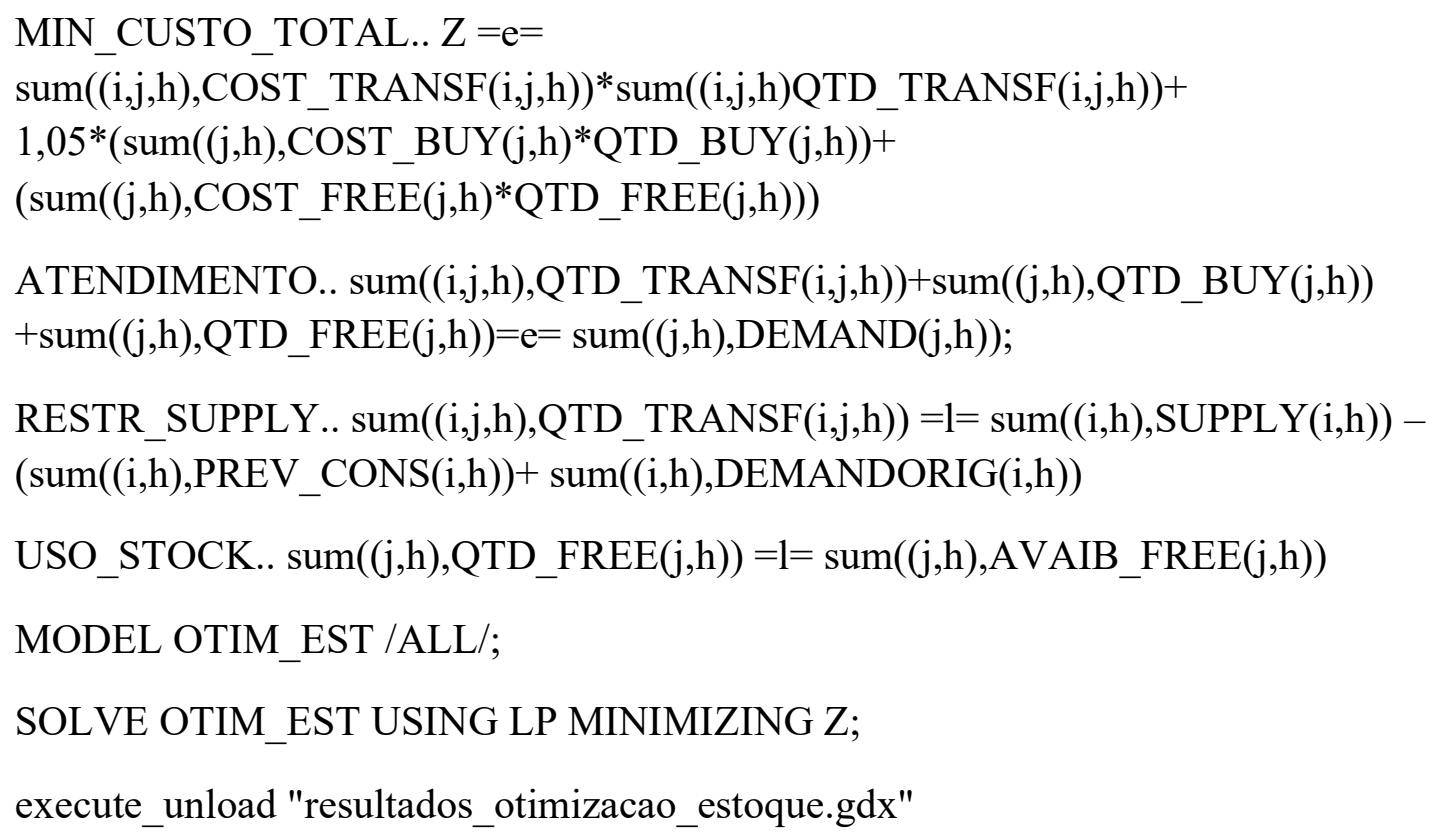

